\title{
Maximum size intersecting families of bounded minimum positive co-degree
}

\author{
József Balogh* Nathan Lemons ${ }^{\dagger} \quad$ Cory Palmer ${ }^{\ddagger}$
}

March 8, 2021

\begin{abstract}
Let $\mathcal{H}$ be an $r$-uniform hypergraph. The minimum positive co-degree of $\mathcal{H}$, denoted by $\delta_{r-1}^{+}(\mathcal{H})$, is the minimum $k$ such that if $S$ is an $(r-1)$-set contained in a hyperedge of $\mathcal{H}$, then $S$ is contained in at least $k$ hyperedges of $\mathcal{H}$. For $r \geq k$ fixed and $n$ sufficiently large, we determine the maximum possible size of an intersecting $r$-uniform $n$-vertex hypergraph with minimum positive co-degree $\delta_{r-1}^{+}(\mathcal{H}) \geq k$ and characterize the unique hypergraph attaining this maximum. This generalizes the Erdös-Ko-Rado theorem which corresponds to the case $k=1$. Our proof is based on the delta-system method.
\end{abstract}

\section{Introduction}

A hypergraph $\mathcal{H}$ is intersecting if for every pair of hyperedges $h, h^{\prime} \in E(\mathcal{H})$ we have $h \cap h^{\prime} \neq \emptyset$. The celebrated theorem of Erdös, Ko and Rado [3] gives that for $n \geq 2 r$, the maximum size of an intersecting $r$-uniform $n$-vertex hypergraph is $\left(\begin{array}{l}n-1 \\ r-1\end{array}\right)$. The Erdös-KoRado theorem is a cornerstone of extremal combinatorics and has many proofs, extensions and generalizations, see the excellent survey of Frankl and Tokushige [11] for a history of extremal problems for intersecting hypergraphs. We call the unique hypergraph achieving the maximum in the Erdős-Ko-Rado theorem a maximal star, i.e., the hypergraph of all hyperedges containing a given vertex.

The degree of a set of vertices $S$ in a hypergraph $\mathcal{H}$ is the number of hyperedges containing $S$, i.e., $|\{h \in E(\mathcal{H}): S \subseteq h\}|$. Denote by $\delta_{s}(\mathcal{H})$ the minimum degree of an $s$-element subset of the vertices of $\mathcal{H}$. In this way, $\delta_{1}(\mathcal{H})$ is the standard minimum degree of a vertex in $\mathcal{H}$.

Huang and Zhao [16] considered a minimum degree version of the Erdős-Ko-Rado theorem. In particular, they proved that for $n \geq 2 r+1$, if $\mathcal{H}$ is an intersecting $r$-uniform

*Department of Mathematics, University of Illinois at Urbana-Champaign, IL, USA, and MIPT, Russian Federation. Email: jobal@illinois.edu. Partially supported by NSF Grant DMS-1764123 and Arnold O. Beckman Research Award (UIUC) Campus Research Board 18132, Simons Fellowship and the Langan Scholar Fund (UIUC).

${ }^{\dagger}$ Theoretical Division, Los Alamos National Laboratory, Email: nlemons@lanl.gov.

${ }^{\ddagger}$ Department of Mathematical Sciences, University of Montana. Email: cory · palmer@umontana.edu. Research supported by a grant from the Simons Foundation \#712036. 
$n$-vertex hypergraph, then $\mathcal{H}$ has minimum degree $\delta_{1}(\mathcal{H}) \leq\left(\begin{array}{l}n-2 \\ r-2\end{array}\right)$. The Huang-Zhao [16] proof uses the linear algebra method and later a combinatorial proof was given by Frankl and Tokushige [10] for $n \geq 3 r$. Kupavskii [19] gave an extension of this result and showed that for $t<r$ and $n \geq 2 r+3 t /(1-t / r)$, every intersecting $r$-uniform $n$-vertex hypergraph $\mathcal{H}$ satisfies $\delta_{t}(\mathcal{H}) \leq\left(\begin{array}{c}n-t-1 \\ r-t-1\end{array}\right)$.

In the more general hypergraph setting, Mubayi and Zhao [22] introduced the notion of co-degree Turán numbers, i.e., the maximum possible value of $\delta_{r-1}(\mathcal{H})$ among all $r$ uniform $n$-vertex hypergraphs $\mathcal{H}$ not containing a specified subhypergraph $\mathcal{F}$. In their paper they give several results that show that the co-degree extremal problem behaves differently from the classical Turán problem.

Motivated by the degree versions of the Erdős-Ko-Rado theorem and co-degree Turán numbers we propose studying the following hypergraph degree condition.

Definition 1. Let $\mathcal{H}$ be a non-empty $r$-uniform hypergraph. The minimum positive codegree of $\mathcal{H}$, denoted $\delta_{r-1}^{+}(\mathcal{H})$, is the maximum $k$ such that if $S$ is an $(r-1)$-set contained in a hyperedge of $\mathcal{H}$, then $S$ is contained in at least $k$ distinct hyperedges of $\mathcal{H}$.

Note that the empty hypergraph is a degenerate case; for simplicity we define its positive co-degree to be zero.

As an example, let us examine hypergraphs that contain no $F_{5}=\{a b c, a b d, c d e\}$ to compare the co-degree and positive co-degree settings. Frankl and Füredi [9] (see [17] for a strengthening) showed that the complete balanced tripartite 3-uniform hypergraph has the maximum number of hyperedges among all 3 -uniform $n$-vertex $F_{5}$-free hypergraphs, for $n$ sufficiently large. This construction has minimum co-degree 0 and it is easy to see that minimum co-degree at least 2 guarantees the existence of an $F_{5}$. On the other hand, the balanced tripartite hypergraph is $F_{5}$-free and has minimum positive co-degree $n / 3$ and it can be shown that minimum positive co-degree strictly greater than $n / 3$ implies the existence of an $F_{5}$.

Note that for ordinary graphs (i.e. 2-uniform hypergraphs), the minimum positive codegree is simply the minimum degree of the non-isolated vertices, which in many extremal problems we may assume is equal to the minimum degree. This suggests positive co-degree as a reasonable notion of "minimum degree" in a hypergraph.

The positive co-degree condition has appeared in several other contexts. For example, in [18] the term $d$-full was used and the authors gave some simple lemmas for hypergraphs with minimum positive co-degree (in the course of proving theorems about extremal numbers for hypergraphs).

In this paper we investigate the maximum size of an intersecting $r$-uniform $n$-vertex hypergraph with positive co-degree at least $k$. As the condition $\delta_{r-1}^{+}(\mathcal{H}) \geq 1$ is vacuous, the maximum in this case is $\left(\begin{array}{l}n-1 \\ r-1\end{array}\right)$ as given by the Erdös-Ko-Rado theorem. The unique construction achieving this bound has minimum positive co-degree 1. On the other hand, as shown in Proposition 4, in an intersecting hypergraph the uniformity gives an upper bound on the minimum positive co-degree, i.e., $r \geq k$. Thus the range of interest for our problem is $2 \leq k \leq r$. In this range we prove that for $n$ sufficiently large the maximumsize intersecting hypergraph with minimum positive co-degree $k$ is given by the following hypergraph.

Definition 2. Given integers $r \geq k \geq 1$ an ( $r$-uniform) $k$-kernel system is a hypergraph 
$\mathcal{H}$ on vertex set $V$ with edges $\mathcal{E}=\left\{E \in\left(\begin{array}{l}V \\ r\end{array}\right):|E \cap X| \geq k\right\}$, were $X$ is a distinguished subset of $V$ of size $2 k-1$. The set $X$ is called the kernel of $\mathcal{H}$.

Clearly a $k$-kernel system is intersecting. Observe that the number of hyperedges in an $r$-uniform $n$-vertex $k$-kernel system $\mathcal{H}$ is

$$
|E(\mathcal{H})|=\sum_{i=k}^{\max \{r, 2 k-1\}}\left(\begin{array}{c}
2 k-1 \\
i
\end{array}\right)\left(\begin{array}{c}
n-2 k+1 \\
r-i
\end{array}\right) \geq\left(\begin{array}{c}
2 k-1 \\
k
\end{array}\right)\left(\begin{array}{c}
n-2 k+1 \\
r-k
\end{array}\right)=\Omega\left(n^{r-k}\right) .
$$

Note that a 1-kernel system is the hypergraph consisting of all hyperedges containing a fixed vertex $x$, i.e., the maximal hypergraph in the Erdős-Ko-Rado theorem. Interestingly, $k$-kernel systems appear as solutions to maximum degree versions of the Erdős-Ko-Rado theorem. Let us give three examples.

First, a special case of a more general theorem of Frankl [7] implies that if $\mathcal{H}$ is a maximum-size intersecting $r$-uniform $n$-vertex hypergraph with maximum degree at most $2\left(\begin{array}{l}n-3 \\ r-2\end{array}\right)+\left(\begin{array}{l}n-3 \\ r-3\end{array}\right)$, then $\mathcal{H}$ is a 2-kernel system, provided $n$ is large enough.

Second, Erdős, Rothschild and Szemerédi (see [2]) posed the following problem: determine the maximum size of an intersecting $r$-uniform $n$-vertex hypergraph $\mathcal{H}$ such that each vertex contained in at most $c|E(\mathcal{H})|$ hyperedges for $r \geq 3$ and $0<c<1$. They proved when $c=2 / 3$ and $n$ large, then a 2-kernel system is the unique hypergraph attaining this maximum. Frankl [5] showed that for $2 / 3 \leq c<1$ and $n$ large enough, $\mathcal{H}$ has no more hyperedges than a 2-kernel system. For $3 / 5<c<2 / 3$ and $n$ large enough, Füredi [5] showed that a 3 -kernel system is one of six non-isomorphic hypergraphs attaining this maximum. In the case when $1 / 2<c \leq 3 / 5$ and $n$ large enough, Frankl [5] showed that $\mathcal{H}$ has no more hyperedges than a 3 -kernel system, although the unique hypergraph attaining this maximum is not isomorphic to a 3-kernel system.

Third, Lemons and Palmer [21] proved that 3-kernel systems are the $r$-uniform $n$ vertex hypergraphs with the largest diversity, i.e., the difference between the number of hyperedges and the maximum degree for $n$ large enough (see [8, 20] for improvements to the threshold on $n$ ).

The main result of our paper is as follows:

Theorem 3. Let $\mathcal{H}$ be an intersecting $r$-uniform $n$-vertex hypergraph with minimum positive co-degree $\delta_{r-1}^{+}(\mathcal{H}) \geq k$ where $1 \leq k \leq r$. If $\mathcal{H}$ has the maximum number of hyperedges, then $\mathcal{H}$ is a k-kernel system for $n$ sufficiently large.

Theorem 3 holds for $n$ large, roughly double exponential in $r$. In Section 3 we give two results that suggest that Theorem 3 should hold for $n$ at least $c r^{k+2}$, where $c$ is a polynomial in $k$. It would be interesting to further refine the range of $n$ as a function of $r$ and $k$ where our results hold. Also, we only considered the positive co-degree of $(r-1)$ sets. We can define $\delta_{s}^{+}(\mathcal{H})$ to be the minimum $k$ such that if $S$ is an $s$-set contained in a hyperedge of $\mathcal{H}$, then $S$ is contained in at least $k$ distinct hyperedges. There may be interesting problems to be considered under this more general condition.

\section{Proof of Theorem 3}

First, let us observe that the uniformity of an intersecting hypergraph is always at least the minimum positive co-degree. 
Proposition 4. If $\mathcal{H}$ is a non-empty intersecting $r$-uniform n-vertex hypergraph with minimum positive co-degree $\delta_{r-1}^{+}(\mathcal{H}) \geq k$, then $r \geq k$.

Proof. Assume, for the sake of a contradiction, that $k>r$. Let $h=\left\{x_{1}, x_{2}, \ldots, x_{r}\right\}$ be a hyperedge of $\mathcal{H}$. The $(r-1)$-set $h \backslash\left\{x_{1}\right\}$ has co-degree at least $k$, so there is a vertex $x_{r+1} \notin h$ such that $\left(h \backslash\left\{x_{1}\right\}\right) \cup\left\{x_{r+1}\right\}$ is a hyperedge of $\mathcal{H}$. Similarly, the $(r-1)$-set $\left(h \backslash\left\{x_{1}, x_{2}\right\}\right) \cup\left\{x_{r+1}\right\}$ has co-degree at least $k$, so there is a vertex $x_{r+2} \notin h \cup\left\{x_{r+1}\right\}$ such that $\left(h \backslash\left\{x_{1}, x_{2}\right\}\right) \cup\left\{x_{r+1}, x_{r+2}\right\}$ is a hyperedge of $\mathcal{H}$. Because $k>r$, we can repeat this process to obtain a hyperedge $\left(h \backslash\left\{x_{1}, \ldots, x_{r}\right\}\right) \cup\left\{x_{r+1}, \ldots, x_{2 r}\right\}=\left\{x_{r+1}, \ldots, x_{2 r}\right\}$ that is in $\mathcal{H}$. Now we have disjoint hyperedges $h$ and $\left\{x_{r+1}, \ldots, x_{2 r}\right\}$ in $\mathcal{H}$ which contradicts the intersecting property.

An $r$-uniform hypergraph $\mathcal{S}$ is a sunflower if every pairwise intersection of the hyperedges is the same set $Y$, called the core of the sunflower. We call the sets $h \backslash Y$ for $h \in E(\mathcal{S})$ the petals of the sunflower $\mathcal{S}$. Note that the petals are pairwise disjoint. Denote the size of the core of a sunflower $\mathcal{S}$ by $c(\mathcal{S})$.

Let $f(r, p)$ denote the minimum integer such that an $r$-uniform hypergraph with $f(r, p)$ hyperedges contains a sunflower with $p$ petals. The Sunflower Lemma of Erdös and Rado [4] claims that $f(r, p) \leq r !(p-1)^{r}$. The determination of $f(r, p)$ is a wellknown open problem in combinatorics. A recent breakthrough by Alweiss, Lovett, $\mathrm{Wu}$ and Zhang [1] gives a bound on $f(r, p)$ of about $(\log r)^{r(1+o(1))}$.

In general we cannot force a sunflower to have a core of a specified size unless we increase the number of hyperedges in the host hypergraph. Mubayi and Zhao (Lemma 6 in [23]) gives conditions for the existence of a sunflower with a core of bounded size.

Lemma 5 (Mubayi and Zhao, [23]). Fix integers $r \geq 3, k \geq 1$ and $p \geq 1$ and let $C=C(r, p)$ be a large enough constant. If $\mathcal{G}$ is an $r$-uniform $n$-vertex hypergraph with

$$
|E(\mathcal{G})| \geq C n^{r-k-1}
$$

then $\mathcal{G}$ contains a sunflower with $p$ petals and core of size at most $k$.

Observe that Lemma 5 is sharp in the order of magnitude of $n$. Indeed, the $r$-uniform $n$-vertex hypergraph consisting of all hyperedges containing a fixed set $Y$ of $k+1$ vertices contains $\left(\begin{array}{l}n-k-1 \\ r-k-1\end{array}\right)$ hyperedges, but no sunflower with a core of size at most $k$ as any two hyperedges intersect in at least $k+1$ vertices. We remark that the problem to determine the best constant $C$ in Lemma 5 is interesting in its own right. In the Appendix at the end of the paper we give a new proof of Lemma 5 that gives an improvement to $C$.

We will need a lower bound on the size of a core of a sunflower in an intersecting hypergraph.

Lemma 6. If $\mathcal{S}$ is a sunflower with at least $r+1$ petals in an intersecting $r$-uniform hypergraph $\mathcal{G}$ with $\delta_{r-1}^{+}(\mathcal{G}) \geq k$, then the core $Y$ of $\mathcal{S}$ satisfies $|Y| \geq k$.

Proof. For the sake of contradiction, assume that the core $Y$ of $\mathcal{S}$ is small, i.e., $|Y|<k$. Observe that $Y$ is a transversal of $\mathcal{G}$, i.e., every hyperedge of $\mathcal{G}$ intersects $Y$. Indeed, as the petals of the sunflower $\mathcal{S}$ are pairwise vertex-disjoint, each hyperedge of $\mathcal{G}$ must intersect the core $Y$ in order to intersect each of the at least $r+1$ hyperedges associated with the petals of the sunflower. 
Now let $Y^{\prime}$ be a minimum transversal in $\mathcal{G}$. Thus $\left|Y^{\prime}\right| \leq|Y|<k$ and the minimality of $Y^{\prime}$ guarantees the existence of a hyperedge $h$ that intersects $Y^{\prime}$ in exactly one element. The $(r-1)$-set $h \backslash Y^{\prime}$ is contained in at most $k-1$ hyperedges of $\mathcal{G}$; one for each element of $Y^{\prime}$. This contradicts the positive co-degree condition on $\mathcal{G}$.

Proof of Theorem 3 , Let $\mathcal{H}$ be an intersecting $r$-uniform $n$-vertex hypergraph with minimum positive co-degree $\delta_{r-1}^{+}(\mathcal{H}) \geq k$ where $1 \leq k \leq r$. Moreover, suppose that $\mathcal{H}$ has the maximum number of hyperedges. We will show that $\mathcal{H}$ is a $k$-kernel system for $n$ sufficiently large.

We have observed that a $k$-kernel system has minimum positive co-degree at least $k$, so we may assume that

$$
|E(\mathcal{H})| \geq\left(\begin{array}{c}
2 k-1 \\
k
\end{array}\right)\left(\begin{array}{c}
n-2 k+1 \\
r-k
\end{array}\right)=\Omega\left(n^{r-k}\right) .
$$

Therefore, for $n$ large enough, Lemmas 5 and 6 guarantees the existence of a sunflower $\mathcal{S}$ with $p=(r+1) r^{k-1}$ petals and core of size $k$. Denote the core of $\mathcal{S}$ by $Y=\left\{y_{1}, y_{2}, \ldots, y_{k}\right\}$.

Note that in order to apply Lemma 5 we need that the following inequality is satisfied:

$$
\left(\begin{array}{c}
2 k-1 \\
k
\end{array}\right)\left(\begin{array}{c}
n-2 k+1 \\
r-k
\end{array}\right) \geq C n^{r-k-1}
$$

where $C=C(r, p)$ is the constant from Lemma 5. This is satisfied when

$$
n \geq \frac{(2 r-2 k)^{r-k}}{\left(\begin{array}{c}
2 k-1 \\
k
\end{array}\right)} C .
$$

The value $C=\left(p r 2^{r}\right)^{2^{r}}$ given in [23] follows from a theorem of Füredi [15].

Claim 7. There is a set of vertices $Z=\left\{z_{1}, z_{2}, \ldots, z_{k-1}\right\}$ such that $Z \cap Y=\emptyset$ and $Z \cup\left\{y_{k}\right\}$ is the core of a sunflower with $r+1$ petals.

Proof. We will prove the following stronger claim: For $0 \leq i \leq k-1$, there is a set of vertices $Z_{i}=\left\{z_{1}, z_{2}, \ldots, z_{i}\right\}$ such that $Y \cap Z_{i}=\emptyset$ and $Z_{i} \cup\left\{y_{k}, y_{k-1}, \ldots, y_{i+1}\right\}$ is the core of a sunflower $\mathcal{S}_{i}$ with $(r+1) r^{k-1-i}$ petals. The claim follows from the case $i=k-1$.

We proceed by induction on $i$. The base case $i=0$ is immediate as $Z_{0}=\emptyset$ and $\mathcal{S}_{0}=\mathcal{S}$ is a sunflower with core $Z_{0} \cup\left\{y_{k}, y_{k-1}, \ldots, y_{1}\right\}=Y$ with $(r+1) r^{k-1}$ petals. Now suppose $i>0$ and the statement holds for $i-1$. Let $\mathcal{S}_{i-1}$ be a sunflower given by the inductive hypothesis.

For each petal $P$ in $\mathcal{S}_{i-1}$ consider the $(r-1)$-set $P \cup Z_{i-1} \cup\left\{y_{k}, \ldots, y_{i+1}\right\}=P \cup$ $Z_{i-1} \cup\left\{y_{k}, \ldots, y_{i}\right\} \backslash\left\{y_{i}\right\}$. By the positive co-degree condition on $\mathcal{H}$, the set $P \cup Z_{i-1} \cup$ $\left\{y_{k}, \ldots, y_{i+1}\right\}$ is contained in $k$ hyperedges of $\mathcal{H}$. Therefore, as $i \leq k-1$, there is a vertex $x(P)$ such that $x(P) \notin\left\{y_{1}, y_{2}, \ldots, y_{i}\right\}$ and $\{x(P)\} \cup P \cup Z_{i-1} \cup\left\{y_{k}, \ldots, y_{i+1}\right\}$ is a hyperedge of $\mathcal{H}$.

Now suppose there are distinct vertices $x_{1}, x_{2}, \ldots, x_{r+1}$ among the vertices in $\{x(P)$ : $P$ is a petal in $\mathcal{S}\}$. Let $P_{1}, P_{2}, \ldots, P_{r+1}$ be the petals corresponding to these vertices, i.e., $\left\{x_{j}\right\} \cup P_{j} \cup Z_{i-1} \cup\left\{y_{k}, \ldots, y_{i+1}\right\} \in E(\mathcal{H})$ for $j=1,2, \ldots, r+1$. Then $Z_{i-1} \cup\left\{y_{k}, \ldots, y_{i+1}\right\}$ is the core of size $k-1$ of a sunflower with petals $P_{j} \cup\left\{x_{j}\right\}$ for $j=1,2, \ldots, r+1$ in $\mathcal{H}$. This contradicts Lemma 6. Therefore, there are at most $r$ distinct vertices among the 
vertices in $\left\{x(P): P\right.$ is a petal in $\left.\mathcal{S}_{i-1}\right\}$. This implies that there is a vertex $x$ that is the vertex $x(P)$ for at least $\frac{1}{r}\left|E\left(\mathcal{S}_{i-1}\right)\right| \geq(r+1) r^{k-2-(i-1)}$ petals $P$ in $\mathcal{S}_{i-1}$. Put $z_{i}=x$ and $Z_{i}=\left\{z_{1}, z_{2}, \ldots, z_{i}\right\}$ and let $\mathcal{S}_{i}$ be the sunflower consisting of $(r+1) r^{k-1-i}$ hyperedges of $\mathcal{S}_{i-1}$ containing $x=z_{i}$. Observe that $Z_{i} \cup\left\{y_{k}, \ldots, y_{i+1}\right\}$ is the core of the sunflower $\mathcal{S}_{i}$ with $(r+1) r^{k-1-i}$ petals.

Let $\mathcal{S}_{Z}$ be a sunflower with $r+1$ petals and core $Z \cup\left\{y_{k}\right\}$ given by Claim 7 . There are at most $(r+1)(r-k)+(k-1)$ vertices disjoint from $Y$ spanned by $\mathcal{S}_{Z}$. As $\mathcal{S}$ has $(r+1) r^{k-1}$ petals, we may choose $r+1$ petals of $\mathcal{S}$ that are vertex-disjoint from the vertices of $\mathcal{S}_{Z}$. Call the resulting sunflower $\mathcal{S}_{Y}$. Note that $\mathcal{S}_{Y}$ has $r+1$ petals and core $Y$.

Claim 8. For every petal $P$ in $\mathcal{S}_{Z}$ and every $y \in Y$ we have that $P \cup Z \cup\{y\}$ is a hyperedge in $\mathcal{H}$.

Proof. Observe that the $(r-1)$-set $P \cup Z$ is contained in the hyperedge $P \cup Z \cup\left\{y_{k}\right\}$, so by the positive co-degree condition $P \cup Z$ is contained in $k$ hyperedges of $\mathcal{H}$. Moreover, each of these hyperedges must intersect every hyperedge in the sunflower $\mathcal{S}_{Y}$. As $\mathcal{S}_{Y}$ has at least 2 petals, each of the $k$ hyperedges containing $P \cup Z$ must contain a distinct vertex of $Y$.

We now continue with a technical claim that will imply the theorem.

Claim 9. For every $k$-set $T \subset Y \cup Z$ we have:

(1) $Q \cup T \in E(\mathcal{H})$ for every petal $Q$ of $\mathcal{S}_{Y}$,

(2) $((Y \cup Z) \backslash T) \cup\{s\} \cup P \in E(\mathcal{H})$ for every $s \in T$ and petal $P$ of $\mathcal{S}_{Z}$.

Proof. We proceed by induction on $t=|T \cap Z|$. Note that $t \leq k-1$. When $t=0$ we have that $T=Y$, then (1) is immediate as $Q \cup Y \in E\left(\mathcal{S}_{Y}\right) \subset \mathcal{H}$ and (2) follows from Claim 8.

Let $t>0$ and suppose the statement of the claim holds for all smaller values of $t$. As $0<t \leq k-1$, there exists a $z \in Z \cap T$ and a $y \in Y \backslash T$. Fix an arbitrary petal $Q$ of $\mathcal{S}_{Y}$. Put $T^{\prime}=T \cup\{y\} \backslash\{z\}$ and note that $\left|T^{\prime} \cap Z\right|=t-1$. Therefore, by induction, we have $Q \cup T^{\prime} \in E(\mathcal{H})$ and $\left((Y \cup Z) \backslash T^{\prime}\right) \cup\left\{s^{\prime}\right\} \cup P \in E(\mathcal{H})$ for every $s^{\prime} \in T^{\prime}$ and petal $P$ of $\mathcal{S}_{Z}$.

By the positive co-degree condition, the $(r-1)$-set $Q \cup T^{\prime} \backslash\{y\}$ is contained in at least $k$ hyperedges. Moreover, $Q \cup T^{\prime} \backslash\{y\}$ is disjoint from the hyperedges of the form $\left((Y \cup Z) \backslash T^{\prime}\right) \cup\{y\} \cup P$ where $P$ is a petal of $\mathcal{S}_{Z}$. As $\mathcal{S}_{Z}$ has $r+1$ petals and $\mathcal{H}$ is intersecting, this implies that the $k$ hyperedges containing $Q \cup T^{\prime} \backslash\{y\}$ each intersect the $k$-set $((Y \cup Z) \backslash T) \cup\{y\}$. In particular, $\left(Q \cup T^{\prime} \backslash\{y\}\right) \cup\{z\}=Q \cup T$ is a hyperedge of $\mathcal{H}$. This proves $(1)$.

In order to prove (2), let us fix an arbitrary petal $P$ of $\mathcal{S}_{Z}$. Observe that the $(r-1)$-set

$$
((Y \cup Z) \backslash T) \cup P=\left((Y \cup Z) \backslash\left(T^{\prime} \cup\{z\} \backslash\{y\}\right)\right) \cup P=\left((Y \cup Z) \backslash T^{\prime}\right) \backslash\{z\} \cup\{y\} \cup P
$$

is contained in the hyperedge $(Y \cup Z) \backslash T^{\prime} \cup\{y\} \cup P \in E(\mathcal{H})$ whose existence is given by the inductive hypothesis on (2) with $y=s^{\prime} \in T^{\prime}$. Therefore, the positive co-degree condition guarantees that the $(r-1)$-set $((Y \cup Z) \backslash T) \cup P$ is contained in $k$ hyperedges. In order for these hyperedges to intersect the $r+1$ hyperedges $Q \cup T$ for each petal $Q$ 
of $\mathcal{S}_{Y}$, we have that each set of the form $((Y \cup Z) \backslash T) \cup\{s\} \cup P$ for $s \in T$ must be a hyperedge of $\mathcal{H}$.

We are now ready to complete the proof of Theorem 3. Suppose that there is a hyperedge $h \in E(\mathcal{H})$ such that $|h \cap(Y \cup Z)| \leq k-1$. Then there exists a $k$-set $T \subset Y \cup Z$ such that $T$ is disjoint from $h$. Moreover, as $\mathcal{S}_{Y}$ has at least $r+1$ petals, there is a petal $Q$ in $\mathcal{S}_{Y}$ that is disjoint from $h$. By Claim 9 we have that $T \cup Q \in E(\mathcal{H})$ which is disjoint from $h \in E(\mathcal{H})$. This violates the intersecting property of $\mathcal{H}$, a contradiction.

Therefore, every hyperedge $h \in E(\mathcal{H})$ intersects $Y \cup Z$ in at least $k$ vertices. This implies that $\mathcal{H}$ is a subhypergraph of a $k$-kernel system, i.e., as $\mathcal{H}$ is edge-maximal, it is exactly a $k$-kernel system.

Remark. Observe that the proof of Theorem 3 gives a stability result. In particular, if $\mathcal{H}$ has enough edges to apply Lemma 5 , then we have that $\mathcal{H}$ is a subhypergraph of a $k$-kernel system.

\section{Improved thresholds on $n$}

We now show that in the case $k \leq 3$, Theorem 3 holds for $n \geq c r^{k+2}$. In Theorem 3 we need $n$ to be at least double exponential in $r$. Recall that two hypergraphs $\mathcal{A}$ and $\mathcal{B}$ are cross-intersecting if for every pair of hyperedges $A \in E(\mathcal{A})$ and $B \in E(\mathcal{B})$ we have $A \cap B \neq \emptyset$. Also, a transversal for a hypergraph $\mathcal{H}$ is a set of vertices $T$ such that $T \cap h \neq \emptyset$ for every hyperedge $h \in E(\mathcal{H})$. The transversal number $\tau(\mathcal{H})$ is the minimum $t$ such that there is a transversal $T$ of $\mathcal{H}$ of size $t$.

We begin with a simple bound on the size of an intersecting hypergraph $\mathcal{H}$ with transversal number $\tau(\mathcal{H})=t$. Stronger results for $\tau(\mathcal{H})=3$ and $\tau(\mathcal{H})=4$ are given by Frankl [6] and Frankl, Ota and Tokushige [12, but we include an argument for the sake of completeness and as our argument holds for all $n$ and $t$.

Lemma 10. Fix $n \geq r \geq t$. Let $\mathcal{H}$ be an intersecting $r$-uniform $n$-vertex hypergraph with transversal number $\tau(\mathcal{H}) \geq t$. Then

$$
|E(\mathcal{H})| \leq r^{t}\left(\begin{array}{l}
n-t \\
r-t
\end{array}\right)
$$

Proof. Let us construct a $t$-uniform hypergraph $\mathcal{T}$ with $|E(\mathcal{T})| \leq r^{t}$ such that for every $h \in E(\mathcal{H})$ there exists a $h^{\prime} \in E(\mathcal{T})$ with $h^{\prime} \subset h$. The existence of $\mathcal{T}$ immediately implies the lemma as $|E(\mathcal{H})| \leq|E(\mathcal{T})|\left(\begin{array}{l}n-t \\ r-t\end{array}\right)$.

We proceed iteratively. First select an arbitrary hyperedge $h_{1} \in E(\mathcal{H})$. For each vertex $v_{1} \in h_{1}$, the set $\left\{v_{1}\right\}$ is not a transversal of $\mathcal{H}$, so there is a hyperedge $h_{2} \in E(\mathcal{H})$ that is disjoint from $\left\{v_{1}\right\}$. For each vertex $v_{2} \in h_{2}$, the set $\left\{v_{1}, v_{2}\right\}$ is not a transversal of $\mathcal{H}$, so there is a hyperedge $h_{3} \in E(\mathcal{H})$ that is disjoint from $\left\{v_{1}, v_{2}\right\}$. We continue this process to select a set of $t$ distinct vertices $v_{1}, v_{2}, \ldots, v_{t}$. Let $\mathcal{T}$ be the collection of all $t$-sets constructed in this way. Note that in each step there are at most $r$ choices for the vertex $v_{i}$, so $|E(\mathcal{T})| \leq r^{t}$.

Now it remains to show that for every $h \in E(\mathcal{H})$ there exists an $h^{\prime} \in E(\mathcal{T})$ with $h^{\prime} \subset h$. Observe that at each step $i$, our hyperedge $h$ must intersect $h_{i}$, so there is 
a choice of vertex in $h_{i} \cap h$. Therefore, there is at least one $r$-set constructed that is contained in $h$.

We first consider the case of minimum positive co-degree at least 2 .

Proposition 11. Fix $r \geq 3$ and let $n \geq \frac{1}{3} r^{4}$. Let $\mathcal{H}$ be an intersecting $r$-uniform $n$-vertex hypergraph with minimum positive co-degree $\delta_{r-1}^{+}(\mathcal{H}) \geq 2$. If $\mathcal{H}$ has the maximum number of hyperedges, then $\mathcal{H}$ is a 2-kernel system.

Proof. We distinguish three cases based on the minimum transversal size $\tau(\mathcal{H})$ of $\mathcal{H}$.

Case 1: $\tau(\mathcal{H})=1$.

Then there is a vertex $x$ in each hyperedge of $\mathcal{H}$. Fix a hyperedge $h \in E(\mathcal{H})$ and observe that the $(r-1)$-set $h \backslash\{x\}$ is contained in exactly one hyperedge which violates the positive co-degree condition.

Case 2: $\tau(\mathcal{H}) \geq 3$.

Then Lemma 10 gives

$$
|E(\mathcal{H})| \leq r^{3}\left(\begin{array}{l}
n-3 \\
r-3
\end{array}\right)
$$

which for $n \geq \frac{1}{3} r^{4}$ is smaller than $3\left(\begin{array}{l}n-3 \\ r-2\end{array}\right)$, a contradiction.

Case 3: $\tau(\mathcal{H})=2$.

Let $\{x, y\}$ be a minimum transversal of $\mathcal{H}$. Consider the $(r-1)$-uniform hypergraphs $\mathcal{H}_{x}=\{h \backslash\{x\}: h \in E(\mathcal{H})$ and $h \cap\{x, y\}=\{x\}\}$ and $\mathcal{H}_{y}=\{h \backslash\{y\}: h \in E(\mathcal{H})$ and $h \cap$ $\{x, y\}=\{y\}\}$. First observe that this pair of hypergraphs is cross-intersecting as $\mathcal{H}$ is intersecting. Now observe that any hyperedge $h \in E\left(\mathcal{H}_{x}\right)$ is a set of size $r-1$ that is contained in a hyperedge of $\mathcal{H}$. Thus, $h$ has co-degree at least 2 , therefore must be a member of $\mathcal{H}_{y}$. This implies that $\mathcal{H}_{x}=\mathcal{H}_{y}$, therefore $\mathcal{H}_{x}$ is intersecting.

Now if $\mathcal{H}_{x}=\mathcal{H}_{y}$ is not a maximal star, then by the Erdös-Ko-Rado theorem we have

$$
|E(\mathcal{H})|<2\left(\begin{array}{l}
n-3 \\
r-2
\end{array}\right)+\left(\begin{array}{l}
n-2 \\
r-2
\end{array}\right)=3\left(\begin{array}{l}
n-3 \\
r-2
\end{array}\right)+\left(\begin{array}{l}
n-3 \\
r-3
\end{array}\right),
$$

i.e., $\mathcal{H}$ has fewer hyperedges than a 2-kernel system, a contradiction. Therefore, every hyperedge of $\mathcal{H}_{x}$ contains a fixed vertex $z$. This implies that every hyperedge of $\mathcal{H}$ contains at least two of $\{x, y, z\}$, i.e., maximality implies that $\mathcal{H}$ is a 2 -kernel system.

We now turn to the case when $k=3$. We will need two lemmas. The first is due to Frankl (Proposition 1.4 in [7]).

Lemma 12 (Frankl, [7]). Let $\mathcal{A}$ and $\mathcal{B}$ be cross-intersecting hypergraphs on vertex set $[N]$ such that $\mathcal{A}$ is a-uniform and $\mathcal{B}$ is $(a+1)$-uniform and intersecting. If $N>2 a+1$, then

$$
|\mathcal{A}|+|\mathcal{B}| \leq\left(\begin{array}{c}
N \\
a
\end{array}\right)
$$

with equality if and only if either $\mathcal{B}$ is empty and $\mathcal{A}$ has size $\left(\begin{array}{l}N \\ a\end{array}\right)$ or both $\mathcal{A}$ and $\mathcal{B}$ are maximal stars containing the same a fixed vertex $q$.

The next lemma gives the size of a minimum transversal for a hypergraph with minimum co-degree at least $k$. 
Lemma 13. Fix $r \geq 3, k \geq 2$ and let $n \geq 2\left(\begin{array}{c}2 k-1 \\ k\end{array}\right)^{-1}(r-k) r^{k+1}$. Let $\mathcal{H}$ be an intersecting $r$-uniform $n$-vertex hypergraph with minimum positive co-degree $\delta_{r-1}^{+}(\mathcal{H}) \geq k$. If $\mathcal{H}$ has the maximum number of hyperedges, then $\mathcal{H}$ has transversal number $\tau(\mathcal{H})=k$.

Proof. First suppose that $\tau(\mathcal{H})<k$. As in the proof of Lemma 6, let $X$ be a minimal transversal for $\mathcal{H}$ and consider a hyperedge $h$ that intersects $X$ in exactly one element. Such a hyperedge exists as otherwise $X$ is not minimal. The $(r-1)$-set $h \backslash X$ is contained in at most $k-1$ hyperedges of $\mathcal{H}$; one for each element of $X$. This contradicts the co-degree condition on $\mathcal{H}$.

Now suppose that $\tau(\mathcal{H})>k$. Lemma 10 gives $|E(\mathcal{H})| \leq r^{k+1}\left(\begin{array}{c}n-k-1 \\ r-k-1\end{array}\right)$. On the other hand, our construction has at least $\left(\begin{array}{c}2 k-1 \\ k\end{array}\right)\left(\begin{array}{c}n-2 k+1 \\ r-k\end{array}\right)$ hyperedges. Therefore, for $n \geq 2\left(\begin{array}{c}2 k-1 \\ k\end{array}\right)^{-1}(r-k) r^{k+1}$ we have a contradiction, thus, $\tau(\mathcal{H})=k$.

Finally, we need a technical definition to construct auxiliary hypergraphs from $\mathcal{H}$.

Definition 14. Let $\mathcal{H}$ be an $r$-uniform hypergraph and let $T$ be a fixed set of vertices in $\mathcal{H}$. For a subset $S \subset T$ define

$$
\mathcal{H}_{S}^{T}=\{h \backslash S: h \in E(\mathcal{H}) \text { and } h \cap T=S\},
$$

i.e., $\mathcal{H}_{S}^{T}$ is the $(r-|S|)$-uniform hypergraph constructed by removing $S$ from each hyperedge of $\mathcal{H}$ that intersects $T$ in exactly $S$.

For ease of notation we will often denote $\mathcal{H}_{S}^{T}$ by $\mathcal{H}_{x_{1} x_{2} \ldots x_{s}}^{T}$ when $S=\left\{x_{1}, x_{2}, \ldots, x_{s}\right\}$.

Theorem 15. Fix $r \geq 3$ and let $n \geq 2 r^{5}$. Let $\mathcal{H}$ be an intersecting $r$-uniform $n$-vertex hypergraph with minimum positive co-degree $\delta_{r-1}^{+}(\mathcal{H}) \geq 3$. If $\mathcal{H}$ has the maximum number of hyperedges, then $\mathcal{H}$ is a 3-kernel system.

Proof. By Lemma 13 we may assume the minimum transversal size of $\mathcal{H}$ is $\tau(\mathcal{H})=3$. Let $X=\{x, y, z\}$ be a minimum transversal of $\mathcal{H}$.

Consider the three $(r-1)$-uniform hypergraphs $\mathcal{H}_{x}^{X}, \mathcal{H}_{y}^{X}$ and $\mathcal{H}_{z}^{X}$. First observe that any pair of these hypergraphs is cross-intersecting as $\mathcal{H}$ is intersecting. Now observe that any hyperedge $h \in E\left(\mathcal{H}_{x}^{X}\right)$ is a set of size $r-1$ that is contained in a hyperedge of $\mathcal{H}$, therefore $h$ has co-degree at least 3. This implies that $h$ is also a member of $\mathcal{H}_{y}^{X}$ and $\mathcal{H}_{z}^{X}$. Thus, all three hypergraphs $\mathcal{H}_{x}^{X}, \mathcal{H}_{y}^{X}, \mathcal{H}_{z}^{X}$ are the same. Moreover, this implies that $\mathcal{H}_{x}^{X}$ is intersecting.

We distinguish three cases based on $\tau\left(\mathcal{H}_{x}^{X}\right)$.

Case 1: $\tau\left(\mathcal{H}_{x}^{X}\right)=1$.

Let $u$ be a transversal of $\mathcal{H}_{x}^{X}$. Every hyperedge of $\mathcal{H}_{x}^{X}, \mathcal{H}_{y}^{X}, \mathcal{H}_{z}^{X}$ contains $u$, therefore, every hyperedge of $\mathcal{H}$ contains at least two vertices from $\{x, y, z, u\}$. Put $T=X \cup\{u\}=$ $\{x, y, z, u\}$.

Claim 16. The six hypergraphs $\mathcal{H}_{a b}^{T}$ for $a, b \in T=\{x, y, z, u\}$ are equal.

Proof. It is enough to show that $E\left(\mathcal{H}_{a b}^{T}\right) \subseteq E\left(\mathcal{H}_{a c}^{T}\right)$ for any three vertices $a, b, c \in T$. Let $h \in E\left(\mathcal{H}_{a b}^{T}\right)$ and consider the $(r-1)$-set $h \cup\{a\}$. By the co-degree condition on $\mathcal{H}$ we have that $h \cup\{a\}$ is contained in at least three hyperedges. Each of these hyperedges includes at least two vertices from $\{x, y, z, u\}$, so $h \cup\{a\}$ is contained in the hyperedge $h \cup\{a, c\}$, i.e., $h \in E\left(\mathcal{H}_{a c}^{T}\right)$. 
Observe that $\mathcal{H}_{x y}^{T}$ and $\mathcal{H}_{z u}^{T}$ are cross-intersecting, which implies that $\mathcal{H}_{x y}^{T}$ is intersecting. Now if $\mathcal{H}_{x y}^{T}$ is not a maximal star, then by the Erdös-Ko-Rado theorem we have

$$
|E(\mathcal{H})|<6\left(\begin{array}{l}
n-5 \\
r-3
\end{array}\right)+4\left(\begin{array}{l}
n-4 \\
r-3
\end{array}\right)+\left(\begin{array}{l}
n-4 \\
r-4
\end{array}\right)=10\left(\begin{array}{l}
n-5 \\
r-3
\end{array}\right)+5\left(\begin{array}{l}
n-5 \\
r-4
\end{array}\right)+\left(\begin{array}{l}
n-5 \\
r-5
\end{array}\right),
$$

i.e., $\mathcal{H}$ has fewer hyperedges than a 3-kernel system, a contradiction. Therefore, every hyperedge of $\mathcal{H}_{x y}$ contains a fixed vertex $v$. As the six hypergraphs $\mathcal{H}_{a b}^{T}$ for $a, b \in$ $T=\{x, y, z, u\}$ are equal, we have that every hyperedge of $\mathcal{H}$ contains at least three of $\{x, y, z, u, v\}$, i.e., maximality implies that $\mathcal{H}$ is a 3 -kernel system.

Case 2: $\tau\left(\mathcal{H}_{x}^{X}\right)=2$.

Let $u, v$ be a minimal transversal of $\mathcal{H}_{x}^{X}$, i.e., every hyperedge of $\mathcal{H}_{x}^{X}$ contains at least one of $u, v$. As $\mathcal{H}_{x}^{X}=\mathcal{H}_{y}^{X}=\mathcal{H}_{z}^{X}$, we have that every hyperedge of $\mathcal{H}$ contains at least two vertices from $T=\{x, y, z, u, v\}$. Moreover, $\mathcal{H}_{x u}^{T}=\mathcal{H}_{y u}^{T}=\mathcal{H}_{z u}^{T}$ and $\mathcal{H}_{x v}^{T}=\mathcal{H}_{y v}^{T}=\mathcal{H}_{z v}^{T}$ and each of these $(r-2)$-uniform hypergraphs is non-empty (as otherwise $u, v$ would not be a minimal transversal). Note that there is no hyperedge that intersects $T$ in exactly $u$ and $v$, so $\mathcal{H}_{u v}^{T}$ is empty. For simplicity, we consider the empty hypergraph as intersecting.

Claim 17. The hypergraph $\mathcal{H}_{a b}^{T}$ is intersecting for every $a, b \in T=\{x, y, z, u, v\}$.

Proof. Suppose not. Then there are hyperedges $A, B \in E\left(\mathcal{H}_{a b}^{T}\right)$ such that $A \cap B=\emptyset$. By the co-degree condition, the $(r-1)$-set $A \cup\{a\}$ is contained in at least three hyperedges of $\mathcal{H}$. Since each hyperedge of $\mathcal{H}$ contains at least two elements from $T$, there is a hyperedge $A \cup\{a, c\}$ where $c \in T \backslash\{a, b\}$. Similarly, the $(r-1)$-set $B \cup\{b\}$ is contained in some hyperedge $B \cup\{b, d\}$ where $d \in T \backslash\{a, b, c\}$. However, the hyperedges $A \cup\{a, c\}$ and $B \cup\{b, d\}$ are disjoint which violates the intersecting property of $\mathcal{H}$.

Now for any $a, b \in T$ we have $\mathcal{H}_{T \backslash\{a, b\}}^{T}$ and $\mathcal{H}_{a b}^{T}$ are cross-intersecting, $\mathcal{H}_{T \backslash\{a, b\}}^{T}$ is $(r-3)$ uniform and $\mathcal{H}_{a b}^{T}$ is $(r-2)$-uniform and intersecting. Therefore, as $n-5>2(r-3)+1$, we may apply Lemma 12 to get

$$
\left|E\left(\mathcal{H}_{a b}^{T}\right)\right|+\left|E\left(\mathcal{H}_{T \backslash\{a, b\}}^{T}\right)\right| \leq\left(\begin{array}{c}
n-5 \\
r-3
\end{array}\right) .
$$

Thus

$$
|E(\mathcal{H})|=\sum_{S \subseteq T}\left|E\left(\mathcal{H}_{S}^{T}\right)\right| \leq 10\left(\begin{array}{l}
n-5 \\
r-3
\end{array}\right)+5\left(\begin{array}{l}
n-5 \\
r-4
\end{array}\right)+\left(\begin{array}{l}
n-5 \\
r-5
\end{array}\right) .
$$

As $\mathcal{H}$ has the maximum number of hyperedges, we must have equality above. Therefore, we must have that for every $a, b \in T$, the hypergraphs $\mathcal{H}_{T \backslash\{a, b\}}^{T}$ and $\mathcal{H}_{a b}^{T}$ have the form of one of the two extremal constructions in Lemma 12. In particular, $\mathcal{H}_{a b}^{T}$ is either empty or a maximal star. As $\mathcal{H}_{x u}^{T}=\mathcal{H}_{y u}^{T}=\mathcal{H}_{z u}^{T}$ and $\mathcal{H}_{x v}^{T}=\mathcal{H}_{y v}^{T}=\mathcal{H}_{z v}^{T}$ are non-empty, each is a maximal star. The hypergraphs $\mathcal{H}_{x u}^{T}$ and $\mathcal{H}_{y v}^{T}$ are cross-intersecting which implies that all six of these these maximal stars share the same fixed vertex $q$. Therefore, we can replace minimal transversal $u, v$ of $\mathcal{H}_{x}^{X}$ with $q$, a contradiction.

Case 3: $\tau\left(\mathcal{H}_{x}^{X}\right) \geq 3$.

Then Lemma 10 gives

$$
\left|E\left(\mathcal{H}_{x}^{X}\right)\right| \leq(r-1)^{3}\left(\begin{array}{c}
(n-1)-3 \\
(r-1)-3
\end{array}\right) \leq r^{3}\left(\begin{array}{c}
n-4 \\
r-4
\end{array}\right) .
$$


The remaining hyperedges of $\mathcal{H}$ are counted by $\mathcal{H}_{x y z}^{X}$ and $\mathcal{H}_{a b}^{X}$ for $a, b \in\{x, y, z\}$. We need a simple claim. Recall that the shadow of an $r$-uniform hypergraph $\mathcal{G}$ is the collection of all $(r-1)$-sets contained in a hyperedge of $\mathcal{G}$. We denote the shadow of $\mathcal{G}$ by $\Delta(\mathcal{G})$.

Claim 18. For each hyperedge $h \in E\left(\mathcal{H}_{y z}^{X}\right)$ there is some hyperedge $g \in E\left(\mathcal{H}_{x}^{X}\right)$ that contains h. Thus,

$$
\left|E\left(\mathcal{H}_{y z}^{X}\right)\right| \leq\left|\Delta\left(\mathcal{H}_{x}^{X}\right)\right| .
$$

Proof. Let $h$ be an arbitrary hyperedge of $\mathcal{H}_{y z}^{X}$. Consider the $(r-1)$-set $A=h \cup\{y\}$. The set $A$ has co-degree at least 3 , so it is contained in three hyperedges of $\mathcal{H}$; one such hyperedge is $A \cup\{z\}$, another could be $A \cup\{x\}$, so there exists at least one hyperedge of the form $A \cup\{w\}$ where $w \notin\{x, y, z\}$. However, $A \cap\{x, y, z\}=\{y\}$, so $(A \cup\{w\}) \backslash\{y\} \in$ $E\left(\mathcal{H}_{y}^{X}\right)=E\left(\mathcal{H}_{x}^{X}\right)$.

By Claim 18 we have

$$
\left|E\left(\mathcal{H}_{y z}^{X}\right)\right| \leq\left|\Delta\left(\mathcal{H}_{x}^{X}\right)\right| \leq(r-1)\left|E\left(\mathcal{H}_{x}^{X}\right)\right| \leq r^{4}\left(\begin{array}{c}
n-4 \\
r-4
\end{array}\right) .
$$

Finally, $\left|E\left(\mathcal{H}_{x y z}^{X}\right)\right| \leq\left(\begin{array}{c}n-3 \\ r-3\end{array}\right)$. Thus,

$$
|E(\mathcal{H})| \leq\left(\begin{array}{l}
n-3 \\
r-3
\end{array}\right)+3\left(r^{4}+r^{3}\right)\left(\begin{array}{l}
n-4 \\
r-4
\end{array}\right)
$$

which is less than $10\left(\begin{array}{c}n-5 \\ r-3\end{array}\right)$ for $n \geq 2 r^{5}$, a contradiction.

In order to extend the technique used in this section to reprove our theorem for minimum positive co-degree $k \geq 4$ we would need to distinguish additional cases based on the transversal size of $\mathcal{H}_{x}^{X}$. Some of these cases can be addressed with Lemmas 10 and 12, but probably new ideas will be needed.

\section{Acknowledgements}

The authors would like to thank Dhruv Mubayi for pointing out reference [23] and Lemma 5. We also thank the anonymous referees for their careful reading of the manuscript and many helpful comments that improved the presentation.

\section{References}

[1] R. Alweiss, S. Lovett, K. Wu and J. Zhang. Improved bounds for the sunflower lemma. STOC 2020: Proceedings of the 52nd Annual ACM SIGACT Symposium on Theory of Computing (2020) 624-630.

[2] P. Erdős. Problems and results in combinatorial analysis. (Italian summary) Colloquio Internazionale sulle Teorie Combinatorie (Rome, 1973), Tomo II, pp. 3-17. Atti dei Convegni Lincei, No. 17, Accad. Naz. Lincei, Rome, 1976.

[3] P. Erdős, C. Ko and R. Rado. Intersection theorems for systems of finite sets. Quart. J. Math. Oxf. 2 (1961) 313-320. 
[4] P. Erdős and R. Rado. Intersection theorems for systems of sets. Journal of the London Mathematical Society 35 (1960) 85-90.

[5] P. Frankl. On intersecting families of finite sets. J. Combinatorial Theory Ser. A 24 (1978) 146-161.

[6] P. Frankl. On intersecting families of finite sets. Bull. Austral. Math. Soc. 21 (1980) $363-372$.

[7] P. Frankl. Erdős-Ko-Rado theorem with conditions on the maximal degree. J. Combin. Theory Ser. A 46 (1987) 252-263.

[8] P. Frankl. Maximum degree and diversity in intersecting hypergraphs. J. Combin. Theory Ser. B 144 (2020) 81-94.

[9] P. Frankl and Z. Füredi. A new generalization of the Erdős-Ko-Rado theorem. Combinatorica 3 (1983) 341-349.

[10] P. Frankl and N. Tokushige. A note on Huang-Zhao theorem on intersecting families with large minimum degree. Discrete Math. 340 (2017) 1098-1103.

[11] P. Frankl and N. Tokushige. Invitation to intersection problems for finite sets. (English summary) J. Combin. Theory Ser. A 144 (2016) 157-211.

[12] P. Frankl, K. Ota and N. Tokushige. Uniform intersecting families with covering number four. J. Combin. Theory Ser. A 71 (1995) 127-145.

[13] Z. Füredi. Erdös-Ko-Rado type theorems with upper bounds on the maximum degree. Algebraic methods in graph theory, Vol. I, II (Szeged, 1978), pp. 177-207, Colloq. Math. Soc. János Bolyai, 25, North-Holland, Amsterdam-New York, 1981.

[14] Z. Füredi. An intersection problem with 6 extremes. Acta Math. Hungar. 42 (1983) $177-187$.

[15] Z. Füredi. On finite set-systems whose every intersection is a Kernel of a star. Discrete Math. 47 (1983) 129-132.

[16] H. Huang and Y. Zhao. Degree versions of the Erdős-Ko-Rado theorem and Erdős hypergraph matching conjecture. J. Combin. Theory Ser. A 150 (2017) 233-247.

[17] P. Keevash and D. Mubayi. Stability theorems for cancellative hypergraphs. J. Combin. Theory Ser. B 92 (2004) 163-175.

[18] A. Kostochka, D. Mubayi and J. Verstraëte. Turán Problems and Shadows I: Paths and Cycles. J. Combin. Theory Ser. A 129 (2015) 57-79.

[19] A. Kupavskii. Degree versions of theorems on intersecting families via stability. J. Combin. Theory Ser. A 168 (2019) 272-287.

[20] A. Kupavskii. Diversity of uniform intersecting families. European J. Combin. 74 (2018) 39-47. 
[21] N. Lemons and C. Palmer. The unbalance of set systems. Graphs Combin. 24 (2008) 361-365.

[22] D. Mubayi and Y. Zhao. Co-degree density of hypergraphs. J. Combin. Theory Ser. A 114 (2007) 1118-1132.

[23] D. Mubayi and Y. Zhao. Forbidding complete hypergraphs as traces. Graphs Combin. 23 (2007) 667-679.

\section{Appendix}

We now give an improvement to Lemma 5 which we believe is of independent interest. Recall that $f(r, p)$ is the minimum integer such that an $r$-uniform hypergraph with $f(r, p)$ hyperedges contains a sunflower with $p$ petals.

Lemma 19. Fix integers $r \geq 3, k \geq 1$ and $p \geq 1$ and let $n$ be large enough. If $\mathcal{G}$ is an $r$-uniform n-vertex hypergraph with

$$
|E(\mathcal{G})| \geq 2 r^{r-k} f\left(r, p r^{r-k}\right)\left(\begin{array}{l}
n-k-1 \\
r-k-1
\end{array}\right),
$$

then $\mathcal{G}$ contains a sunflower with p petals and core of size at most $k$.

This replaces the value of $C=\left(p r 2^{r}\right)^{2^{r}}$ in Lemma 5 with $C=2 r^{r-k} f\left(r, p r^{r-k}\right)$ which is significantly smaller when using the bound on $f\left(r, p r^{r-k}\right)$ from [1].

Proof. For the sake of a contradiction, suppose that $\mathcal{G}$ contains no sunflower with $p$ petals and core of size at most $k$.

Iteratively remove from $\mathcal{G}$ a sunflower $\mathcal{S}$ with exactly $\operatorname{pr}^{c(\mathcal{S})-k}$ petals such that at each step we choose a sunflower with minimum available core size $c(\mathcal{S})$. Let $t$ be the number of steps in this sunflower removal procedure. Note that $t$ grows with $n$ as at each step we remove at most $p r^{r-k}$ hyperedges from $\mathcal{G}$ and we only need constant number of hyperedges to guarantee the existence of a sunflower with $\operatorname{pr}^{c(\mathcal{S})-k}$ petals. In particular, we have

$$
t \geq \frac{|E(\mathcal{G})|-f\left(r, p r^{r-k}\right)}{p r^{r-k}} \geq \frac{|E(\mathcal{G})|}{2 p r^{r-k}}
$$

for $n$ large enough.

The core of each removed sunflower is of size at least $k+1$ and at most $r-1$. Therefore, there is some integer $s$ such that there are at least $t / r$ cores of size $s$ among the removed sunflowers. Some of these cores may be identical. Let us compute the maximum multiplicity of a core $Y$. There are at most $\left(\begin{array}{l}n-|Y| \\ r-|Y|\end{array}\right)$ hyperedges containing $Y$ and each removed sunflower with core $Y$ has exactly $p^{|Y|-k}$ hyperedges. Therefore, the maximum multiplicity of a core $Y$ is at most

$$
\frac{1}{p r^{|Y|-k}}\left(\begin{array}{c}
n-|Y| \\
r-|Y|
\end{array}\right) \leq \frac{1}{p r}\left(\begin{array}{c}
n-k-1 \\
r-k-1
\end{array}\right)
$$


for $n \geq r$. Therefore, there is a collection of at least

$$
(t / r) p r\left(\begin{array}{l}
n-k-1 \\
r-k-1
\end{array}\right)^{-1} \geq p \frac{|E(\mathcal{G})|}{2 p r^{r-k}}\left(\begin{array}{c}
n-k-1 \\
r-k-1
\end{array}\right)^{-1} \geq f\left(r, p r^{r-k}\right)
$$

distinct cores of size $s$. Let $Y_{1}, Y_{2}, \ldots, Y_{q}$ be these cores and let $\mathcal{S}_{i}$ be the sunflower with core $Y_{i}$ for $i=1,2, \ldots, q$. Note that each of these sunflowers has exactly $\mathrm{pr}^{s-k}$ petals.

Let $t$ be the first step in the sunflower removal procedure in which a sunflower with core of size $s$ is chosen to be removed. This implies that all later cores are of size at least $s$. Now we will show that there is a sunflower $\mathcal{B}$ with core of size less than $s$ and $\operatorname{pr}^{c(\mathcal{B})-k}$ petals among the hyperedges in the sunflowers $\mathcal{S}_{1}, \mathcal{S}_{2}, \ldots, \mathcal{S}_{q}$. Before removing the sunflower in step $t$, all hyperedges of the sunflowers $\mathcal{S}_{1}, \mathcal{S}_{2}, \ldots, \mathcal{S}_{q}$ are still in $\mathcal{H}$. Therefore, the sunflower $\mathcal{B}$ with core of size less than $s$ could be chosen in step $t$, this will contradict the choice of $t$.

We may think of the $s$-sets $Y_{1}, \ldots, Y_{q}$ as an $s$-uniform hypergraph on the vertex set of $\mathcal{H}$. As $q \geq f\left(r, p r^{r-k}\right) \geq f\left(s, p r^{r-k}\right) \geq f\left(s, p r^{s-k}\right)$, the $s$-sets $Y_{1}, \ldots, Y_{q}$ contain an $s$-uniform sunflower $\mathcal{A}$ with $p r^{s-k}$ petals and core $Y^{*}$ of size less than $s$. By relabelling, we may suppose that $Y_{i}$ is a member of $\mathcal{A}$ for $i=1,2, \ldots, p r^{s-r}$. Note that the petals $Y_{i} \backslash Y^{*}$ of $\mathcal{A}$ are pairwise disjoint by definition. The sunflower $\mathcal{A}$ is not in the hypergraph $\mathcal{H}$ as it is $s$-uniform. However, each hyperedge of $\mathcal{A}$ is the core of some sunflower $\mathcal{S}_{i}$ in $\mathcal{H}$. Therefore, we will use the members of $\mathcal{A}$ to identify an $r$-uniform sunflower $\mathcal{B}$ with core $Y^{*}$ in $\mathcal{H}$. The main idea will be carefully choose a petal from each sunflower $\mathcal{S}_{i}$ whose core is a member of $\mathcal{A}$. To this end, define $\mathcal{B}$ as follows:

First pick any hyperedge of $\mathcal{S}_{1}$; denote it by $h_{1}$. Now suppose we have chosen $\ell$ hyperedges $h_{1}, h_{2}, \ldots, h_{\ell}$ that form a sunflower with core $Y^{*}$. The union of these hyperedges contains $\ell\left(r-\left|Y^{*}\right|\right)$ vertices outside of $Y^{*}$. Therefore, as long as

$$
p r^{s-k}>\ell\left(r-\left|Y^{*}\right|\right)
$$

there is a petal $Y_{i} \backslash Y^{*}$ of $\mathcal{A}$ that is disjoint from each of the hyperedges $h_{1}, h_{2}, \ldots, h_{\ell}$. The corresponding sunflower $\mathcal{S}_{i}$ with core $Y_{i}$ has

$$
p r^{s-k}>\ell\left(r-\left|Y^{*}\right|\right)
$$

petals by (1). Therefore, there is a petal $P$ of $\mathcal{S}_{i}$ that is also disjoint from the hyperedges in $h_{1}, h_{2}, \ldots, h_{\ell}$. Let $h_{\ell+1}$ be the hyperedge $P \cup Y_{i}$. Now we have a sunflower with $\ell+1$ petals and core $Y^{*}$. We may repeat this procedure as long as $\ell$ satisfies (1), i.e., until $\ell=p r^{s-k-1}$. This implies that the number of petals in sunflower $\mathcal{B}$ is at least

$$
p r^{s-k-1} \text {. }
$$

As $\mathcal{B}$ has core $Y^{*}$ of size $c(\mathcal{B})<s$ we have a contradiction to the choice of sunflower in step $t$. 